\title{
Study on the Evaluation for the Knowledge Sharing Efficiency of the Knowledge Service Network System in Agile Supply Chain
}

\author{
Wang Daoping ${ }^{1}$, Wang Zhe ${ }^{1, *}$ and Sheng Xiaojuan ${ }^{2}$ \\ ${ }^{1}$ Donlinks School of Economics and Management, University of Science and Technology, Beijing, 100083, P.R. China; \\ ${ }^{2}$ Management School, Beijing Union University, Beijing, 100101, P.R. China
}

\begin{abstract}
The agility of supply chain as well as the competitiveness of the knowledge service network system is affected by knowledge sharing efficiency. In this paper, an evaluation index system for the knowledge sharing efficiency of the knowledge service network system in agile supply chain was constructed in terms of knowledge system, knowledge sharing environment, coordinating actions and sharing results. After evaluation indexes were reduced with rough set theory, a knowledge sharing efficiency evaluation model for the knowledge service network system in agile supply chain was established with error back propagation (BP) artificial neural network, and the network model was simulated with sample data, proving the evaluation result could comprehensively, accurately reflect the actual situation. Thus, the model possesses a very broad application prospect.
\end{abstract}

Keywords: Agile supply chain, artificial neural network, efficiency, knowledge service network system, knowledge sharing.

\section{INTRODUCTION}

The knowledge service network system in agile supply chain is a well-connected network built by the knowledge service members and demand members in agile supply chain, in order to provide a knowledge service. In this network, service members are driven by the instant knowledge demands of agile supply chain members to excavate and create valuable, dynamic information resources and also share them on the knowledge service platform, so as to provide all sorts of intelligence supports and services demanded by the members of agile supply chain. Knowledge sharing efficiency is a relative index to measure the degree of closeness between the real knowledge sharing results and the maximum expectation in the knowledge sharing process of the network members, while the agility of supply chain as well as the overall competitiveness of the knowledge service network system is affected by this degree. In the knowledge service network system in agile supply chain, the relationship between the factors affecting knowledge sharing and the knowledge sharing efficiency is a complex cross-impact, which is focused by many researchers. For example, Matzler and others [1] analyzed the impact of employee personality characteristics on knowledge sharing efficiency; Glomseth and others [2] discussed the impact from the form of organizational culture on knowledge sharing efficiency; Yang [3] illustrated that the cooperative organizational culture and instructional, creative leadership played a role in promoting knowledge sharing efficiency using cases; Siemsen and others [4] analyzed the sharing behavior efficiency of knowledge sharing subjects in terms of sharing motivation, time, and ability; Wen [5], following earlier study by Wang [6] and others [7-9] analyzed the relationship of the characteristics of organizational structure, the form of organizational culture, and the interaction of internal units with knowledge sharing through case study.

In existing document literatures, however, the impact of one or several factors on sharing efficiency is only studied. In the knowledge service network system, in fact, the members in knowledge sharing and the entire network organization are in a complex dynamic system. There are also many factors affecting knowledge sharing efficiency. Thus, these document literatures are difficult to quantify and reflect knowledge sharing as well as the complex law of its efficiency. In this paper, a non-linear relationship between knowledge sharing efficiency and its affecting factors was studied by building an artificial neural network model from the perspective of system theory.

\section{AN EVALUATION INDEX SYSTEM FOR THE KNOWLEDGE SHARING EFFICIENCY OF THE KNOWLEDGE SERVICE NETWORK SYSTEM IN AGILE SUPPLY CHAIN}

Knowledge sharing in the knowledge service network system in agile supply chain exists between the enterprises in the same agile supply chain, which is a type of crossorganizational activities and also much more complex than the internal sharing activities of an enterprise. It aims to effectively share knowledge resources. The particularities of knowledge resources make the evaluation on the knowledge sharing efficiency in the supply chain difficult to implement and quantify, etc. The key to effectively evaluate the knowledge sharing efficiency in the supply chain lies in the establishment of a scientific and reasonable evaluation index system. To choose each index for the evaluation index system, 
Table 1. Knowledge sharing efficiency evaluation index system of knowledge service network in agile supply chain.

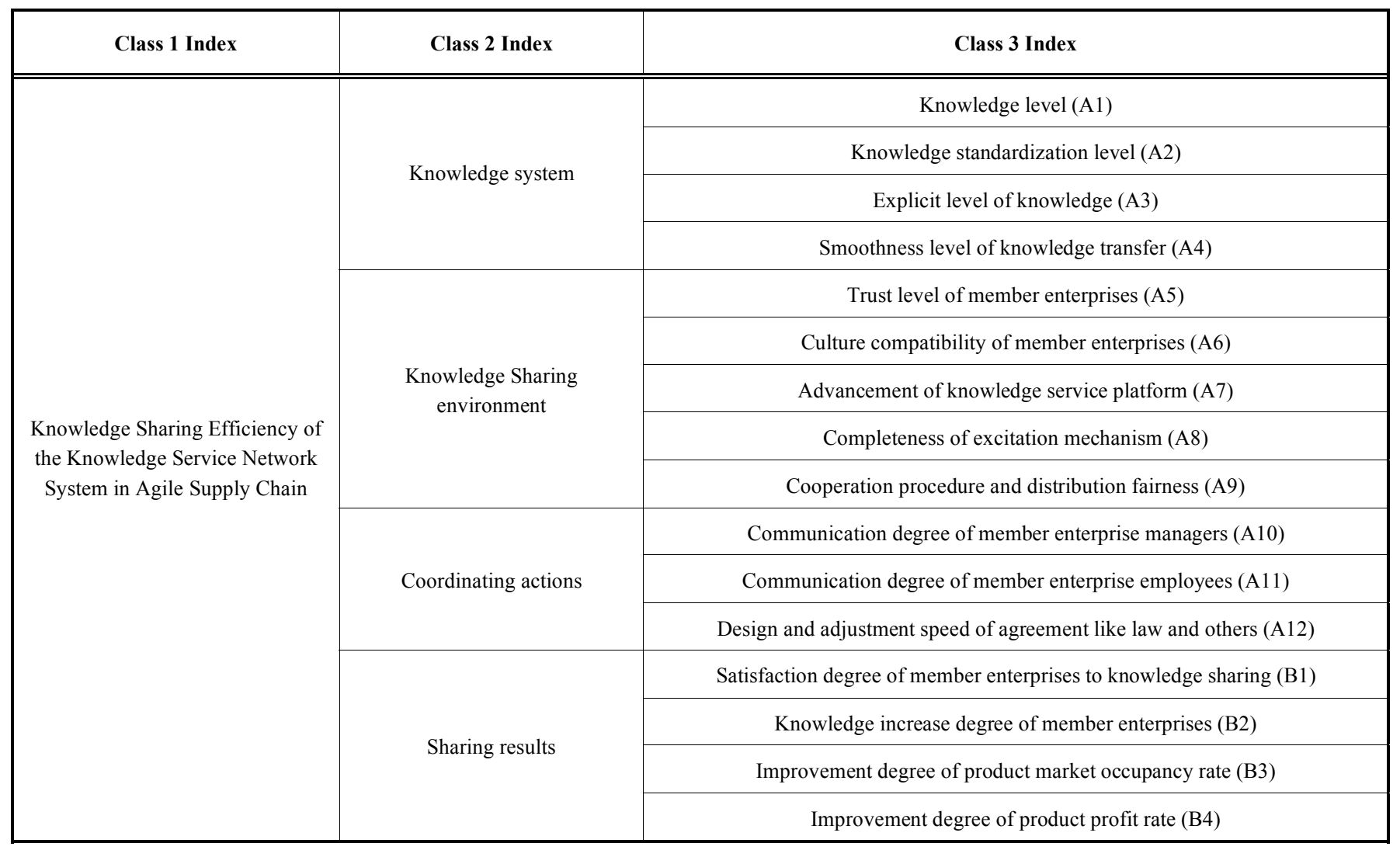

the factors in both knowledge resources and agile supply chain management were necessarily considered, which should be reflected in the evaluation index system.

The system included the indexes directly and indirectly affecting the knowledge sharing efficiency of the knowledge service network system in agile supply chain. 16 indexes were designed mainly in terms of knowledge system, knowledge sharing environment, coordinating actions and sharing results [10]. The established index system is as shown Table 1.

The index system was specially designed for the knowledge sharing in the knowledge service network system in agile supply chain. First, the interaction and coordination between the network members were highlighted, because these activities were the sources to produce knowledge sharing efficiency. Second, the information exchange and learning between the network members were emphasized. The members could know well the cognitive gap between each other and narrow it in the process of knowledge sharing to achieve a cooperative effect, only if they shared valuable knowledge and information through communication and coordination. Third, the self-organizing, self-learning and dynamic evolution characteristics of the network organizations were underlined. When the network members were in mutual coordination and learning, the network organizations could give a full play the self-organizing and self-learning characteristics and constantly adjust the knowledge sharing environment through dynamic evolution, so as to promote a harmonious and healthy development for the knowledge sharing environment.

\section{THE EVALUATION MODEL FOR THE KNOWL- EDGE SHARING EFFICIENCY OF THE KNOWL- EDGE SERVICE NETWORK SYSTEM IN AGILE SUPPLY CHAIN}

\subsection{Training Sample Collection and Basic Analysis}

To collect training samples, a questionnaire approach was applied and the questionnaire design was divided into two parts. Its main part included all the indexes of the evaluation index system for the knowledge sharing efficiency of the knowledge service network system in agile supply chain. Likert Scale was used for measuring the questionnaire. Thus, five points from low to high were set up for $\mathrm{A} 1 \sim \mathrm{A} 12$ and $\mathrm{B} 1 \sim \mathrm{B} 4$ of the index system according to the test objects' discriminability: 1-the lowest; 2-low; 3-ordinary; 4-high; 5-the highest. On this basis, 40 questionnaires were distributed to the industry-related, different-sized companies, and 35 of them were returned, so the rate of return was $87.5 \%$.

Next, the reliability and validity of the investigated data were analyzed with SPSS. Reliability analysis was to calculate the reliability coefficient of each secondary index and the overall reliability coefficient of the questionnaire, so as to test the internal consistency and reliability of the questionnaire data. The reliability coefficient of each index and the overall reliability coefficient of the questionnaire were estimated mainly with Cronbach's $\alpha$.

From Table 2, Cronbach's $\alpha$ coefficient of the four secondary indexes of the questionnaire was $>0.7$ and the overall Cronbach's $\alpha$ coefficient of the questionnaire was $>0.9$, indi- 
Table 2. Reliability index of investigation questionnaire.

\begin{tabular}{|c|c|c|c|c|c|}
\hline Reliability Index & Knowledge System & Knowledge Sharing Environment & Coordinating Actions & Sharing Results & Questionnaire \\
\hline \hline Cronbach's $\alpha$ & 0.755 & 0.728 & 0.71 & 0.773 & 0.913 \\
\hline
\end{tabular}

cating the reliability of the questionnaire met the requirements.

Validity analysis included content validity analysis and construct validity analysis. Content validity referred to the coverage of the contents in the designed questionnaire for the study subject, in which expert evaluation was usually applied. The questionnaire about knowledge sharing efficiency was obtained through literature reading and actual research. To make the questionnaire clear and complete in contents and good in content validity, some questions were revised repeatedly after the first draft was finished. Thus, the contents necessary to measure were fully covered by the questionnaire. Construct validity was tested with factor analysis, and the results of KMO and Bartlett Test of Sphericity was as shown in Table 3.

Table 3. Results of KMO test and bartlett test of sphericity.

\begin{tabular}{|c|c|c|}
\hline \multicolumn{2}{|c|}{ Kaiser-Meyer-Olkin Test } & $\mathbf{. 8 3 0}$ \\
\hline \hline \multirow{3}{*}{ Bartlett Test of Sphericity } & Approximate Chi-Square & 270.168 \\
\cline { 2 - 3 } & Degree of Freedom (df) & 120 \\
\cline { 2 - 3 } & Significant Level (Sig.) & .000 \\
\hline
\end{tabular}

From Table 3, KMO test value was 0.830 (the significance level was $<0.05$ ), and the data was suitable for factor analysis, and a significantly construct and correlation existed between the original variables, so a good construct validity was available.

\subsection{Rough Set Reduction Indexes}

Rough set theory is a mathematical tool to process fuzzy and uncertain knowledge, which is applicable to solving the qualitative index screening problem in knowledge sharing efficiency evaluation [11]. Its main idea is to equivalently, effectively simplify the set of attributes under the condition of ensuring it meet certain classification accuracy and implement the simplified calculation by removing redundant attributes. Rough set needs no prior attribute distribution information for the reduction of the attributes, and its application results were more objective. Therefore, rough set can very well solve the qualitative evaluation index screening problem in knowledge sharing efficiency evaluation [12].

According to the basic steps of rough set to screen evaluation indexes, 12 indexes (A1 A12) in the evaluation index system for the knowledge sharing efficiency of the knowledge service network system in agile supply chain were used as condition attributes; B was as the set of decision attributes and represented the comprehensive evaluation result of knowledge sharing efficiency, which was obtained by rounding the arithmetic average of B1 B4 sample data in the sharing result of evaluation index system; a decision table of index attributes was finally constructed with condition attributes and decision attributes, as shown in Table 4. The data in the table was discrete in itself, and thus the samples were not discretely processed.

The indexes were reduced using the genetic algorithm of Rosetta data processing software, and the values of all parameters were as follows: crossover probability was 0.3 ; exchange and mutation probability was 0.05 ; reverse mutation probability was 0.05 ; the population size was 70.5 indexes were reduced, and 7 indexes (A1, A3, A4, A5, A8, A10, \& A11) were ultimately retained.

\subsection{The Artificial Neural Network Modeling and Simula- tion}

Artificial neural network is a new information processing or computation system to imitate the structure or function of the biological nervous system based on the basic features of the brain. The large number of information processing units constitutes a dynamic information processing system through

Table 4. The decision table of index attributes.

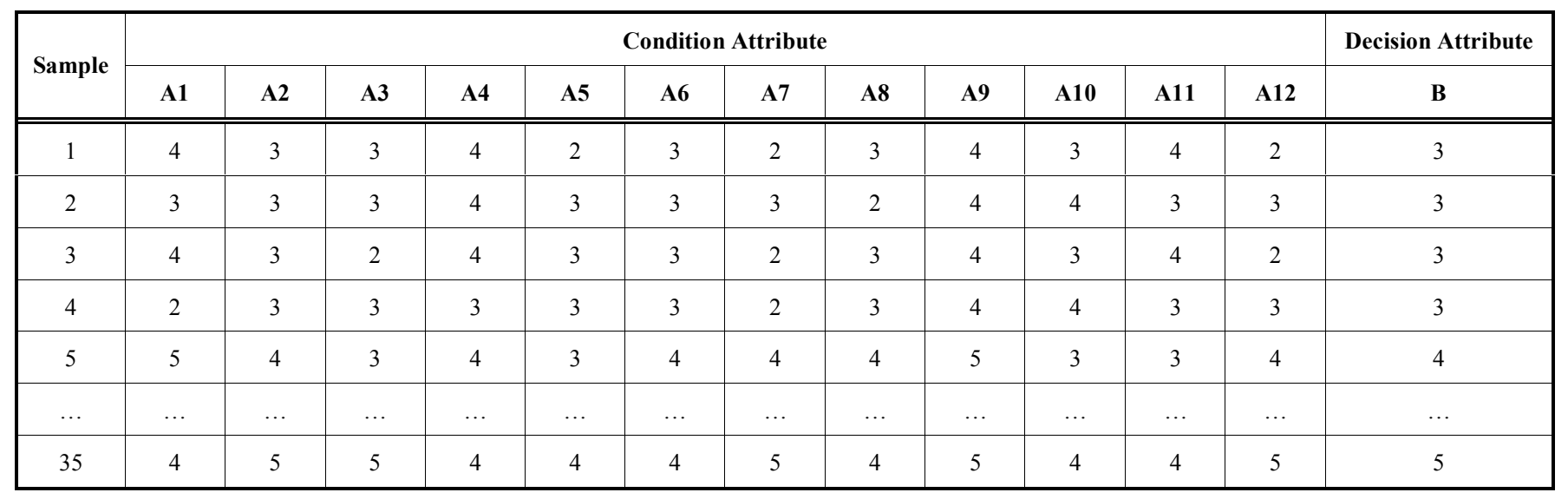




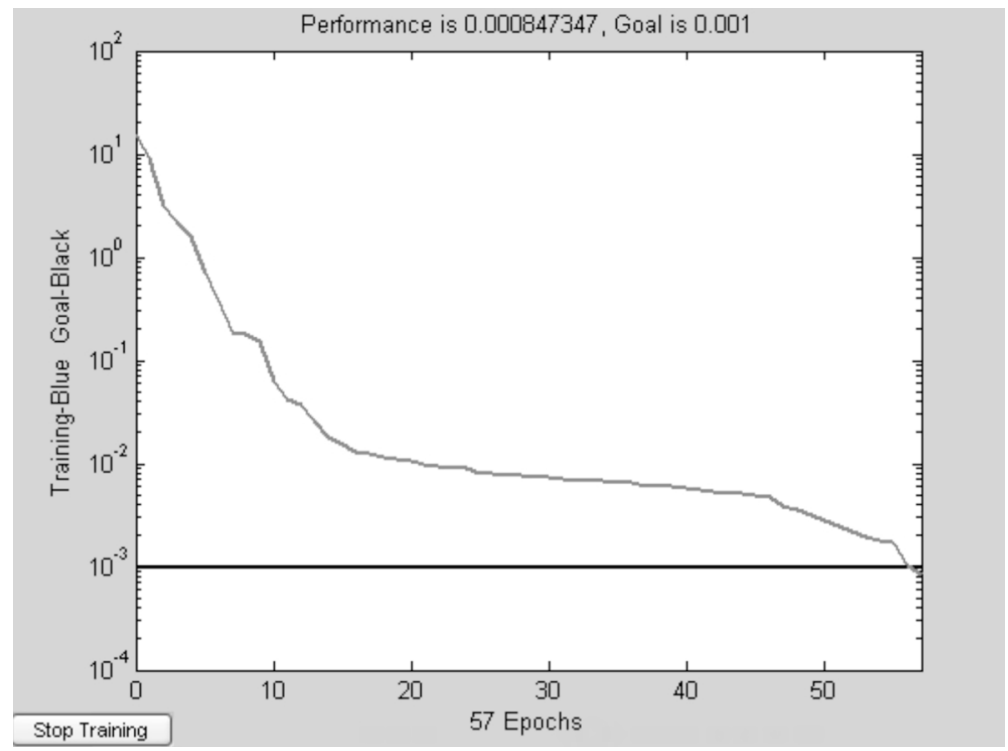

Fig. (1). The neural network training error changing curve.

Table 5. The simulation results of knowledge sharing efficiency.

\begin{tabular}{|c|c|c|c|c|}
\hline Sample & B1 & B2 & B3 & B4 \\
\hline \hline 31 & 3.9980 & 3.016318 & 2.9459 \\
\hline 32 & 4.0048 & 3.9202 & 4.0581 & 4.0028 \\
\hline 33 & 4.0264 & 4.0047 & 4.9463 & 4.0759 \\
\hline 34 & 4.1912 & 5.0216 & 4.4118 & 3.7501 \\
\hline 35 & 4.0037 & 2.9720 & 4.0198 & 3.9738 \\
\hline
\end{tabular}

an extensive connection, from which the complex, fuzzy, dynamic, random, and nonlinear relationships between multiple factors can be reflected [13, 14].

In 1985, the parallel distributed processing group under the guidance of Rumelhart et al proposed an error backpropagation (BP algorithm). BP network is a hierarchical network structure with three or above layers, in which the neurons between adjacent lower and upper layers are fully connected [15]. That is, a weighting link is between each neuron of the upper layer and each neuron of the lower layer, but neurons in each layer are not linked. In neural network, the rule to amend weight is referred to as the learning algorithm. The learning process of BP is composed of forwardpropagating and backward-propagating. In forwardpropagating, input information is propagated into the output layer from the input layer via the hidden layer, and the state of the neurons in each layer affects that of next layer. It will be backward-propagated and the error signal returns along the original neuron pathway, if it is not output as expected from the output layer. In the returning, the weight of neuron connection is revised one by one. This process is constantly iterative and finally will make the signal error into a permitted range [16].

Under Matlab7.0 environment, the first 30 samples in the sample data simplified with rough set were used as training samples, and the last samples were as test samples for data analysis. In the training of artificial neural network model, a three-layer neural network training model was established using the training sample data as the input sample set of artificial neural network and B1 B4 as the set of corresponding output samples. In the model, the dimension of input layer, output layer, and hidden layer was respectively 7, 4, and 11; the value of learning efficiency was 0.1 ; error precision was 0.001 . After 57 iterative trainings, the requirement for error termination was fulfilled, and thus a desired BP artificial neural network model was obtained, as shown in Fig. (1).

The test samples were simulated using well-trained BP artificial neural network model, as shown in Table 5. The knowledge sharing efficiency simulation results of 5 samples were compared with the actual survey data. In Fig. (2), the consistency between the simulation output and the actual survey data was good, indicating the model fitting effect was good.

\section{CONCLUSION}

The study on the knowledge sharing activities in the knowledge service network system found that artificial neural network has many advantages to evaluate knowledge sharing efficiency compared with the subjective evaluation by the traditional evaluators. First, the weights of artificial neural network model are obtained through learning, which can reflect the nonlinear relationship between indexes and 


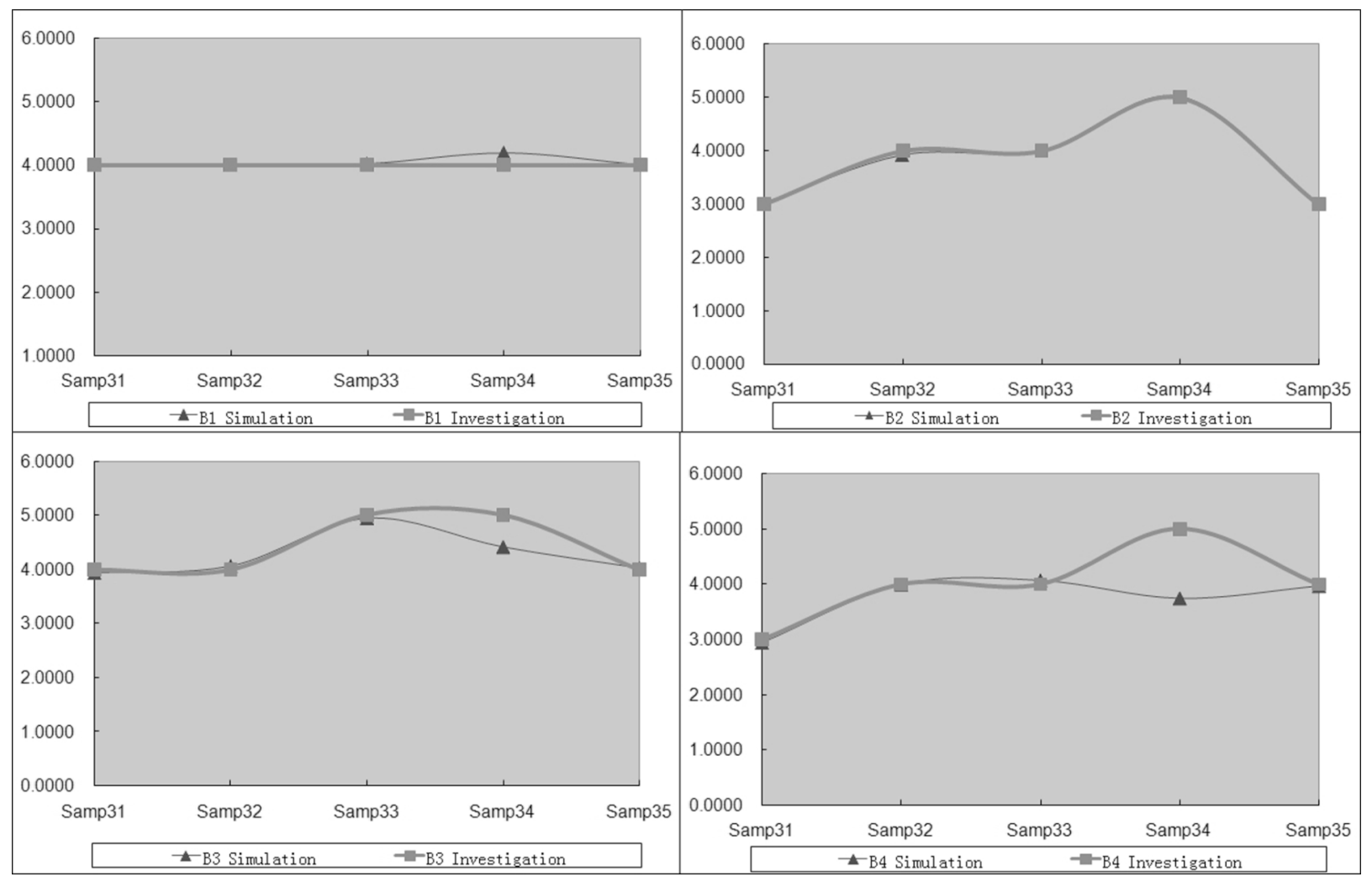

Fig. (2). Comparison between knowledge sharing efficiency simulation results and actual survey data.

knowledge sharing efficiency and avoid the human factors and uncertainties in the previous expert grading method. Second, artificial neural network possesses unique selforganizing, self-learning and strong fault tolerant ability and discover new rules by classifying learning data as knowledge sharing efficiency evaluation involves many factors and is very complex, for making the evaluation system more intelligent. Finally, artificial neural network evaluation method features fast calculating speed, high fitting precision and strong operability, and can improve the efficiency of evaluation and also make the simulation results comprehensively reflect to the actual situation when it is applied to evaluate the knowledge sharing efficiency of the knowledge service network system in agile supply chain. Thus, a theoretical basis and practical reference is provided for a better knowledge sharing of the knowledge service network system in agile supply chain.

\section{CONFLICT OF INTEREST}

The authors confirm that this article content has no conflict of interest.

\section{ACKNOWLEDGEMENTS}

Declared none.

\section{REFERENCES}

[1] K. Matzler, B. Renzl, J. Müller, S. Herting, and T.A. "Mooradian, Personality traits and knowledge sharing", Journal of Economic Psychology, vol. 29, no. 3, pp. 301-313, 2008.

[2] R. Glomseth, P. Gottschalk, and H. Solli-Saether, "Occupational culture as determinant of knowledge sharing and performance in police investigations", International Journal of the Sociology of Law, vol. 35, no. 2, pp. 96-107, 2007.

[3] J. Yang, "Knowledge sharing: investigating appropriate leadership roles and collaborative culture", Tourism Management, vol. 28, no. 2, pp. 530-543, 2007.

[4] E. Siemsen, A.V. Roth, and S. Balasubramanian, "How motivation, opportunity, and ability drive knowledge sharing: the constrainingfactor model", Journal of Operations Management, vol. 26, no. 3, pp. 426-445, 2008.

[5] W. Lin, "The effect of knowledge sharing model", Expert Systems with Applications, vol. 34, no. 2, pp. 1508-1521, 2008.

[6] S. Wang, and R.A. Noe, "Knowledge sharing: a review and directions for future research", Human Resource Management Review, vol. 20, no. 2, pp. 115-131, 2010.

[7] M. Friesl, S.A. Sackmann, and S. Kremser, "Knowledge sharing in new organizational entities: the impact of hierarchy, organizational context, micro-politics and suspicion", Cross Cultural Management: An International Journal, vol. 18, no. 1, pp. 71-86, 2011.

[8] P. Liu, B. Raahemi, and M. Benyoucef, "Knowledge sharing in dynamic virtual enterprises: a socio-technological perspective", Knowledge-Based Systems, vol. 24, no. 3, pp. 427-443, 2011.

[9] X. Gu, Q. Wei, and C. Yu, "Inter-organizational similarity as a driver of knowledge sharing within supply chains", Proceedings of the $8^{\text {th }}$ International Conference on Management Science and Engineering Management, Springer, US, pp. 665-676, 2014.

[10] C. Feng, T. Li, and Y. Han, "Research on performance evaluation of knowledge sharing in supply chain", Science of Science and Management of S. \& T, vol. 5, pp. 12, 2011.

[11] S. Greco, B. Matarazzo, and R. Slowinski, "Rough sets theory for multicriteria decision analysis", European Journal Of Operational Research, vol. 129, no. 1, pp. 1-47, 2001.

[12] K. Zhang, and G.T. Chi, "Establishment of ecological evaluation indicators system based on correlation analysis-rough set theory", Journal of Systems Engineering, vol. 27, pp. 119-128, 2012.

[13] A. Goh, "Back-propagation neural networks for modeling complex systems", Artificial Intelligence in Engineering, vol. 9, no. 3, pp. 143-151, 1995.

[14] P. Zheng, and J. Li, "Back propagation neural network approach on supply chain dynamic performance measurement", Operations Research and Management Science, vol. 2, pp. 7, 2010. 
[15] A.J. Trappey, C.V. Trappey, T. Chiang, and Y. Huang, "Ontologybased neural network for patent knowledge management in design collaboration", International Journal of Production Research, vol. 51, no. 7, pp. 1992-2005, 2013.
[16] X. Fan, S. Zhang, L. Wang, Y. Yang, and K. Hapeshi, “An evaluation model of supply chain performances using 5DBSC and LMBP neural network algorithm", Journal of Bionic Engineering, vol. 10, no. 3, pp. 383-395, 2013.

Received: September 16,2014

Revised: December 23, 2014

Accepted: December 31, 2014

(C) Daoping et al.; Licensee Bentham Open.

This is an open access article licensed under the terms of the Creative Commons Attribution Non-Commercial License (http://creativecommons.org/licenses/by-nc/3.0/) which permits unrestricted, non-commercial use, distribution and reproduction in any medium, provided the work is properly cited. 